\title{
Results of open reduction internal fixation versus percutaneous iliosacral screw fixation for unstable pelvic ring injuries: retrospective study of 36 patients
}

\author{
Sami Abou-Khalil ${ }^{1} \cdot$ Sylvain Steinmetz $^{1} \cdot$ Laurent Mustaki $^{1} \cdot$ Bertrand Leger $^{2} \cdot$ Eric Thein $^{1} \cdot$ Olivier Borens $^{1}$
}

Received: 24 December 2019 / Accepted: 27 February 2020

(c) Springer-Verlag France SAS, part of Springer Nature 2020

\begin{abstract}
Introduction Surgical stabilization of posterior pelvic ring fractures can be achieved by closed reduction and percutaneous fixation (CRPF) or by open reduction and internal fixation (ORIF). The aim of the present study is to compare the clinical results of both methods.

Material and methods Medical records of 36 patients consecutively operated for unstable pelvic ring injuries were retrospectively reviewed. We compared 22 patients treated with CRPF versus 14 patients stabilized by using ORIF between 2007 and 2017. The Majeed and Pohlemann scores were used to evaluate postoperative functional outcomes. Complications like blood loss, infection rate, Neurological injury, the operative time and the length of hospital stay were analyzed.

Results The median Majeed pelvic score was 87 points for the CRPF technique compared with 69 points for the ORIF technique. The median Pohlemann score, operative time and length of hospitalization were similar between the two groups. The median blood loss for the CRPF technique was $300 \mathrm{ml}$ compared to $500 \mathrm{ml}$ for the ORIF technique. CRPF and ORIF procedure had each one neurological lesion. There was one case of infection in the ORIF group and none in the CRPF group. No measurements except for the blood loss have reached the significance threshold.

Conclusion The CRPF technique shows a clear decrease in blood loss. There was no statistically significant difference in the functional results, infection rate, neurological injury, operative time and hospital stay between both techniques.
\end{abstract}

Keywords Percutaneous iliosacral screw fixation · Unstable pelvic ring injury · Iliosacral fracture/dislocation · Majeed score $\cdot$ Pohlemann score

Sylvain Steinmetz

sylvain.steinmetz@chuv.ch

Sami Abou-Khalil

sami.aboukhalil@chuv.ch

Laurent Mustaki

laurent.mustaki@chuv.ch

Bertrand Leger

Bertrand.leger@crr-suva.ch

Eric Thein

eric.thein@chuv.ch

Olivier Borens

olivier.borens@chuv.ch

1 Department of Orthopaedic Surgery, Centre Hospitalier Universitaire Vaudois (CHUV), Rue du Bugnon 46, 1011 Lausanne, Switzerland

2 Institute for Research in Rehabilitation, Clinique Romande de Réadaptation Suvacare, Avenue Grand-Champsec 90, 1950 Sion, Switzerland

\section{Introduction}

Posterior pelvic ring injuries are complex, often unstable, and difficult to treat. Sacroiliac fractures are a variant of pelvic injury associated with high energy. One of the multiple factors associated with poor functional outcomes is residual posterior displacement [1]. The goal of operative treatment for displaced, unstable pelvic ring fractures and sacroiliac dislocations is anatomic reduction and rigid fixation to facilitate early mobilization.

Depending on the type of fracture, there are different surgical approaches, reduction techniques, and fixation methods. Examples of fixation methods include iliosacral screws, trans-sacral screws, sacral bars, vertebropelvic internal fixation, posterior tension band plating, and anterior sacroiliac (SI) joint plating.

Pelvic fractures can be reduced in an open or closed fashion followed by percutaneous and/or internal fixation. Open 
reduction and internal fixation (ORIF) with anterior plating have traditionally required extensive surgical exposure of the pelvic deep structures which can be associated with nerve damage [2, 3], elevated surgical blood loss [2] and infection rates of about $25 \%$ [4].

Posterior plating risks include wound complications with deep infection reported to be between 14 and $25 \%[2,4,5]$.

Closed reduction and percutaneous fixation (CRPF) has been recommended by Routt et al. and has demonstrated good results [6]. Malreduction of the pelvic ring can lead to significant long-term problems like pain, non-union or malunion with leg length discrepancy and delayed neurological compromise [2]. Iliosacral or trans-sacral screw fixation of pelvic ring fractures can be technically demanding procedures with the use of an intraoperative simple 2D fluoroscopically assisted imaging, or with a CT navigated system.

Routt el al. described the CRPF technique for sacroiliac fractures and dislocations with an iliosacral screw in the first sacral vertebral body demonstrating it to be biomechanically stable [7]. This method allows also a reduction in operating time [8], diminished surgical bleeding [8] and decreased infection rate ranging from 0 to $1 \%$ [9].

Our primary outcome is the functional results of ORIF and CRPF for posterior pelvic ring injuries using the Majeed [10] and Pohlemann [11] scores. Secondary outcomes analysis include complications (blood loss, infection rate, and Neurological injury), operative time and length of hospitalization.

\section{Material and methods}

The present retrospective study was conducted between September 2007 and December 2017. During this time, fifty patients sustained an unstable posterior pelvic ring injury at an academic level-1 trauma center.

Of these 50 patients, 36 adult patients were treated with iliosacral fixation. The patients were classified into two groups: the CRPF group had 22 patients stabilized under fluoroscopic (59\%) or O-Arm guidance (41\%) and the ORIF group had 14 patients.

The median follow-up was 6 months for the CRPF group and 10.5 months for the ORIF group (Table 1).

One patient in the CRPF group and one patient in the ORIF group were lost to follow-up.

Fourteen patients had to be excluded due to the following: patients under 18 years of age, patients whose initial posterior stabilization used a method other than CRPF and ORIF, H-shaped sacral fractures, and those treated only with external fixation.

Fractures of the pelvis were classified according to the AO-Tile classification [2]. In the CRPF group, 19 patients had a Tile $\mathrm{C}$ and 3 patients a Tile $\mathrm{B}$ fracture. In the ORIF group, 8 patients were classified according to Tile $\mathrm{C}$ and 6 according to Tile B (Table 1).

The median age was 35.5 years with 25 th -75 th percentiles $(27 ; 59)$ in the CRPF group versus 42.5 years median age with 25 th-75th percentiles $(26 ; 54)$ in the ORIF group. The gender distribution was similar between the two groups
Table 1 Patients characteristics. Assessments of CRPF and ORIF techniques. Median (IQR)

\begin{tabular}{lccc}
\hline & CRPF $(n=22)$ & ORIF $(n=14)$ & $p$ value \\
\hline Age (years), median (IQR) & $35.5(27 ; 59)$ & $42.5(26 ; 54)$ & 0.85 \\
Female sex & $4(18 \%)$ & $2(14 \%)$ & 0.50 \\
Type B2/3 AO-Tile classification & $3(14 \%)$ & $6(43 \%)$ & - \\
Type C AO-Tile classification & $19(86 \%)$ & $8(57 \%)$ & - \\
Majeed score (points), median (IQR) & $87(59 ; 96)^{\mathrm{c}}$ & $69(64 ; 86)^{\mathrm{a}}$ & 0.23 \\
Majeed (percent of good to excellent results) & $15(71 \%)^{\mathrm{c}}$ & $6(46 \%)^{\mathrm{a}}$ & 0.14 \\
Pohlemann score (points), median (IQR) & $5(4 ; 6)^{\mathrm{c}}$ & $5(3 ; 6)^{\mathrm{a}}$ & 0.74 \\
Pohlemann (percent of fair to excellent results) & $15(71 \%)^{\mathrm{c}}$ & $9(69 \%)^{\mathrm{a}}$ & 0.89 \\
Operative time (min) & $139(93 ; 179)^{\mathrm{d}}$ & $138(116 ; 160)$ & 0.82 \\
Duration of hospitalization (days) & $23(13 ; 32)$ & $25(20 ; 41)$ & 0.31 \\
Blood loss (ml) & $300(100 ; 450)^{\mathrm{e}}$ & $500(240 ; 1000)^{\mathrm{b}}$ & 0.03 \\
Infections & $0(0 \%)^{\mathrm{c}}$ & $1(7 \%)$ & 0.40 \\
Neurological injury after surgery & $1(5 \%)^{\mathrm{c}}$ & $1(7 \%)^{\mathrm{c}}$ & 0.76 \\
Neurological injury related to trauma & $8(38 \%)^{\mathrm{c}}$ & $3(23 \%)^{\mathrm{c}}$ & 0.36 \\
Follow-up (months) & $6(4 ; 12)$ & $10.5(7 ; 12)$ & 0.07 \\
Radiological score (points) & $2(1 ; 3)$ & $2(2 ; 3)$ & 0.33 \\
Anterior approach and fixation & $16(71 \%)$ & $6(38 \%)$ & 0.07 \\
Anterior pelvic injury & $22(100 \%)$ & $13(92 \%)$ & 0.20 \\
\hline
\end{tabular}

${ }^{\mathrm{a}} n=13 ;{ }^{\mathrm{b}} n=11 ;{ }^{\mathrm{c}} n=21 ;{ }^{\mathrm{d}} n=20 ;{ }^{\mathrm{e}} n=17$ 
with $18 \%$ female in the CRPF group and $14 \%$ in the ORIF group (Table 1).

\section{Surgical techniques}

Two experienced orthopedic surgeons (OB and ET), specialists in pelvic and acetabular surgery, performed all the procedures.

ORIF technique was favored in patients with crescent fractures and when closed manipulative reduction failed. Over the last years, most of the patients had a percutaneous fixation as our surgeons acquired experience and familiarity with this technique.

A single dose of antibiotic prophylaxis (1.5 g of cefuroxime i.v.) was given in all the cases.

\section{Closed (CRPF)}

The patient was placed supine on a radiolucent table, and an image intensifier was moved between inlet, outlet and lateral views to facilitate reduction and screw placement. Closed reduction was performed by longitudinal traction and closed manipulation. Reduction was held manually, while cannulated, percutaneous iliosacral screws were placed. All screws were partially threaded and used with washers. Moderate compression was applied across the SI joint, but in the case of vertical sacral fractures, screws were tightened only until the fracture surfaces were approximated.

The posterior lesions were fixed with Depuy Synthes ${ }^{\circledR}$ $7.3 \mathrm{~mm}$ cannulated cancellous screws (San Juan Capistrano, California, United States) (Fig. 1).

The anterior pelvic ring injuries were fixed with $3.5 \mathrm{~mm}$ curved stainless steel R75 pubic symphysis plates Stryker ${ }^{\circledR}$.
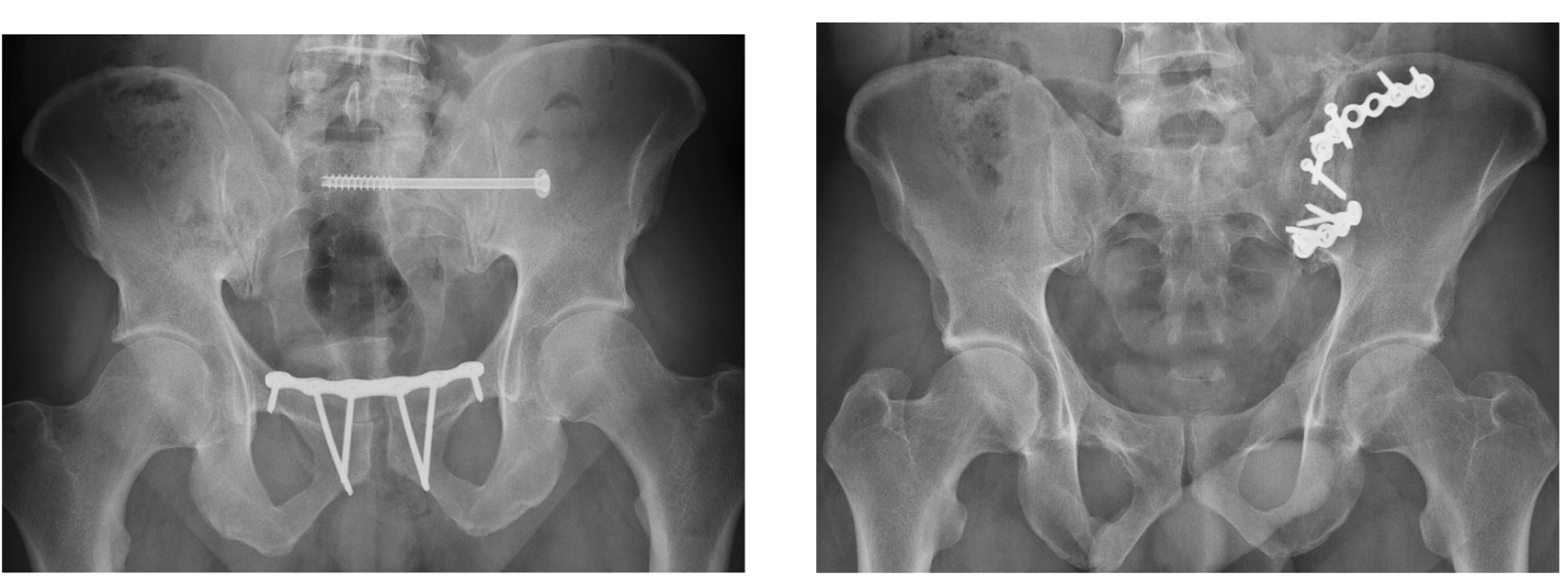

Fig. 1 CRPF technique

\section{Open (ORIF)}

The open reduction technique involved supine and prone positioning on a radiolucent fracture table. A gluteus maximus sparing method for the posterior approach was used to allow for direct visualization and reduction of the posterior pelvic injury.

Intraoperative fluoroscopy was used to confirm reduction and guide the placement of iliosacral screws.

In ORIF technique, the posterior pelvic ring injuries were fixed with $3.5 \mathrm{~mm}$ Matta pelvic plates Stryker $^{\circledR}, 5 \mathrm{~mm}$ condyle screws with a nut of a T2 femoral nailing system Stryker ${ }^{\circledR}$, Depuy Synthes ${ }^{\circledR} 3.5 \mathrm{~mm}$ reconstruction plates DCP, $3.5 \mathrm{~mm}$ locking compression plates LCP, bolted sacral bars, and $7.3 \mathrm{~mm}$ cannulated cancellous screws (San Juan Capistrano, California, United States) (Fig. 2).

The anterior pelvic ring injuries were fixed with 3.5 curved stainless steel R75 pubic symphysis plates Stryker ${ }^{\circledR}$.

\section{The postoperative assessment}

The Majeed Score is based on pain, work performance, sitting, sexual intercourse and standing. The total score ranks the clinical outcomes as excellent ( $>85)$, good (70-84), acceptable $(55-69)$ or poor $(<55)$ in patients who were working before the injury [10]. The Pohlemann score is divided into clinical, radiological, and social reintegration outcomes [11]. For radiological results, we measured vertical, antero-posterior and symphyseal displacements on the last antero-posterior radiographic view of the pelvis using the technique described by Henderson et al. [12]. The measurements were performed by three distinct observers.

For the posterior displacement, the Pohlemann radiological score was rated as three points for anatomical posterior healing, two points for maximal posterior displacement of
Fig. 2 ORIF technique 
$5 \mathrm{~mm}$ and one point for posterior displacement above $5 \mathrm{~mm}$ [11].

To evaluate the pelvic outcome, the ratings of the radiological and clinical results were assessed as a single score on a 7 points scale, where the maximum of 7 points represented an excellent result, 6 points a good result, 5 and 4 points a fair result, and 3 and 2 points a bad result [11].

The complications (blood loss, infection, and neurological injury), operative time, and length of hospitalization were analyzed.

\section{Statistical analysis}

Descriptive statistics were used to characterize the population. Continuous variables were expressed as the median and interquartile range (IQR) [25th-75th percentiles]. Categorical variables were summarized as a percentage.

Chi-squared tests were used to assess significant differences between observed frequencies. Wilcoxon signedrank tests were used to compare scores and measurements between both groups of patients. All statistical analyses were performed with Stata 15.0 (StataCorp, College Station, TX, USA). The limit for statistical significance was set as usual at $p<0.05$.

\section{Results}

In the CRPF group, 18 patients were fixed with a single screw in S1, 2 patients with two screws in S1, 1 patient with a screw in $\mathrm{S} 1$ and $\mathrm{S} 2$, and 1 patient with one screw in $\mathrm{S} 1$ and $\mathrm{S} 3$.

Twenty-two patients of the CRPF group had an anterior pelvic injury $(p=0.07)$. Sixteen of them required a simultaneous anterior approach and fixation $(p=0.20)$ (Table 1).

In the ORIF group, eleven patients were fixed with pelvic plates, one patient with one screw in $\mathrm{S} 1$ and $\mathrm{S} 2$, one patient with sacral bars, one patient with 2 condylar screws of a distal femoral nail.

Thirteen patients of the ORIF group had an anterior pelvic injury $(p=0.07)$. Four of them required a simultaneous anterior approach and fixation, whereas two of these patients were fixed few days later $(p=0.20)$ (Table1).

\section{The functional scores outcomes}

Majeed and Pohlemann scores were obtained between April and September 2018 in 21 patients for the CRPF group and 13 patients for the ORIF group. The median follow-up for the CRPF group was 6 months with 25 th-75th percentiles $(4 ; 12)$. The median follow-up for the ORIF group was 10.5 months with 25 th-75th percentiles $(7 ; 12)$ (Table 1$)$.
The median Majeed score was 87 points with 25th-75th percentiles $(59 ; 96)$ for the CRPF group compared to median score of 69 points with 25 th-75th percentiles $(64 ; 86)$ for the ORIF group (Table 1). The percentage of good to excellent results with the Majeed score was $71 \%$ for the CRPF technique against $46 \%$ for the ORIF technique.

The median Pohlemann score was 5 points with 25th-75th percentiles $(4 ; 6)$ for the CRPF group compared to a median score of 5 points with 25 th-75th percentiles (3; 6) for the ORIF group (Table 1). The percentage of fair to excellent results with the Pohlemann score was $71 \%$ for the CRPF technique against $69 \%$ for the ORIF technique.

The results did not reach significant threshold.

\section{Radiological score}

The median radiological score was 2 points for the CRPF group with 25 th-75th percentiles $(1 ; 3)$ and 2 points for the ORIF technique with 25 th-75th percentiles $(2 ; 3)$. The $p$ value $(p=0.33$ ) was not significant (Table 1$)$.

\section{Blood loss}

Sixteen of the twenty-two patients treated with the CRPF method had simultaneous anterior fixation and four of the fourteen patients treated with the ORIF method had simultaneous anterior fixation. The CRPF group had a median blood loss of $300 \mathrm{ml}$ with 25 th-75th percentiles $(100 ; 450)$ compared to $500 \mathrm{ml}$ with 25 th-75th percentiles $(240 ; 1000)$ for the ORIF group. The $p$ value $(p=0.03)$ was significant (Table 1).

\section{Infection}

There was one case of infection in the ORIF technique out of fourteen patients and none in the CRPF technique. The $p$ value $(p=0.40)$ was not significant (Table 1$)$.

\section{Neurologic lesions}

For neurological injuries, the CRPF technique had one lesion versus one lesion for the ORIF technique. The $p$ value $(p=0.76)$ was not significant (Table 1$)$.

With the CRPF method, one patient had neuropathic pain in the territory of S1 and S3 due to malpositioning of the screws. After changing the screws, the patient fully recovered without sensory or motor loss.

With the ORIF method, the neurologic complication was due to a malpositioned guide wire in the L5 nerve root causing a permanent steppage gait requiring an orthosis. 


\section{Operative time and length of hospitalization}

The median operative time was 139 min with 25 th-75th percentiles $(93 ; 179)$ for the CRPF technique versus $138 \mathrm{~min}$ with 25 th-75th percentiles $(116 ; 160)$ for the ORIF technique. The $p$ value $(p=0.82)$ was not significant (Table 1$)$.

The two cases in the CRPF group with unilaterally posterior-only fixation procedures had an operative time of $47 \mathrm{~min}$ and $56 \mathrm{~min}$.

The median length of hospitalization was 23 days with 25th-75th percentiles $(13 ; 32)$ for CRPF technique compared to 25 days with 25 th-75th percentiles $(20 ; 41)$ for ORIF technique. The $p$ value $(p=0.31)$ was not significant (Table 1).

\section{Discussion}

Unstable pelvic injuries have been associated with high rates of morbidity and mortality [5]. The operative management of unstable pelvic ring fractures can be technically difficult and necessitates accurate reduction with rigid fixation. The treatment method is an important factor that can influence the functional outcomes [1]. Optimal treatment for unstable fractures of the pelvis is not yet defined [13]. The ORIF technique includes anterior and posterior approaches. The anterior approach to the SI joint with subsequent ORIF provides better exposure of the anterior SI joint with a direct visualization of the injury in some cases. However, it is difficult to assess reduction in pure SI joint dislocations through this approach. The ideal assessment of a reduced SI joint dislocation is via the posterior approach and determination at the region of the inferior SI joint near the greater sciatic notch. With this anterior approach, injury to the L5 nerve root is a risk and the medial access is limited to apply a plate.

The sacroiliac screw is a well-recognized choice of implant for posterior stabilization of pelvic injuries. Fixation was most commonly carried out using an open posterior approach until recently with the development of the percutaneous approach [6]. CRPF technique is certainly a more difficult and demanding method that requires experienced surgeons because of the particularity of pelvic and sacral anatomy [14] with a risk of injury to the cauda equina, L5 and $\mathrm{S} 1$ nerve roots.

\section{Functional scores}

Very few studies have compared the functional results of the two techniques. Elzohairy et al. retrospectively reviewed 70 patients treated with CRPF and ORIF. Using the Pohlemann score, he found no significant difference in the functional outcomes between the two methods with good to excellent results in $86 \%$ of cases for the CRPF against $80 \%$ for the ORIF [15].

In our study, we also found no significant difference in the functional outcomes with the Pohlemann score in both techniques. We didn't obtain as much as good to excellent results than M.M. Elzoheiry et al.; however, we had 86\% and $57 \%$ of Tile C pelvic fractures for the CRPF andORIF technique compared to $26 \%$ for both techniques in their study [15].

Based on the Majeed's grading score for pelvic fractures, we had better functional outcomes using the CRPF technique with a Majeed's median score of 87 points compared to 69 points for the ORIF technique. We found $71 \%$ of good to excellent results with the CRPF group versus $46 \%$ with the ORIF group.

Schweitzer et al. [9] obtained better functional outcomes using the CRPF method with $91 \%$ of good to excellent results.

The main limitation of this score is that it is influenced by the characteristics of the fracture and associated injuries and not determined by the stabilization method [9].

Various authors affirm that good functional results after stabilization of sacroiliac lesions depends on the quality of the posterior reduction $[4,11,12,16,17]$.

We found in our study a better quality of reduction in the ORIF technique with $42.8 \%$ anatomical reduction according to the Pohlemann radiological score compared to $27.2 \%$ in the CRPF technique.

Anatomic reconstruction of the pelvic ring is an important factor for good to excellent clinical results, but even when this goal is met, other parameters (sacral fractures, sacroiliac dislocations, primary neurological/urological injuries) can lead to an unsatisfactory result [18].

We observed also in our study that some patients in the CRPF group had good or excellent clinical results despite the poor quality of reduction.

Of the twenty-two patients, eight had a radiologic score of 1 point. Five of them had good to excellent results with the Majeed outcome scale and six of them had fair results using the Pohlemann score.

These outcomes correlate with other studies were the concept of bad functional results and non-anatomical reduction of the posterior pelvic injury has been questioned [19].

Controversy still remains regarding the factors that affect the functional result in the treatment of unstable pelvic ring fractures [1]. Several studies like Suzuki et al. and Kokubo et al. showed that nerve damage has a significant relationship with unsatisfactory functional outcome $[1,20]$

We did not find in our study a direct relationship between bad results and neurologic lesions.

Seven out of nine patients in the CRPF group with operative and non-operative neurologic injuries had good to 
excellent results according to the Majeed score. The other two patients had poor results.

In the ORIF group, three out of five patients had good to excellent results. One patient had a fair result and the other patient had a poor result.

According to the Pohlemann score, the nine patients with associated neurologic injuries had fair results with the CRPF technique knowing that four of them had a radiologic score of 1 point which tends to decrease the overall score.

For the ORIF technique, three out four patients had fair results with a radiologic score of 2 points for two of them. The fourth patient had an excellent result.

The majority of the patients treated by CRPF and ORIF method with the worst Majeed and Pohlemann functional outcomes were young males ( 21 to 44 years old) with associated traumatic injuries (brain, thoracic, abdominal, upper and lower extremities).

In the CRPF technique, the three patients had $\mathrm{C} 2$ fractures without neurological lesions. Two of them had associated injuries and a radiological score of 1 point. The other patient had no associated injury and had a radiological score of 2 points.

In the ORIF technique, the two patients had a B3 fracture with associated injuries. One of them had a traumatic neurological lesion. Both patients had associated injuries. One patient had a radiologic score of 1 point and the other had a radiological score of 3 points.

\section{Blood loss}

The disadvantage of the ORIF technique includes a major risk of bleeding due to the extensive surgical exposure to reach deep structures. The percutaneous technique popularized by Routt in the 1990s resulted in a decreased blood loss $[8,21]$. The main factors are the lack of decompression of the intrapelvic hematoma and the decrease in intraoperative bleeding [22]. Our study confirms these observations with blood loss reduced by nearly half using the percutaneous technique knowing that the bleeding amount was probably overestimated in the CRPF group due to the simultaneous anterior approach and fixation (71\%).

\section{Infection}

Percutaneous sacroiliac screw fixation seems to be an attractive option for the infection rate. In the literature, the infection rate is undoubtedly high in the open method with reports of up to $25 \%$ [4]. In the ORIF technique, we found a rate of infection of $7.1 \%$ and none in the CRPF. These results for the CRPF technique correspond to the infection rate between 0 and $1 \%$ described in the Schweitzer et al. publication [9].

\section{Neurologic lesions}

One of the potential disadvantages of percutaneous technique is the proximity of neurovascular structures, especially L5-S1 and iliac vessels [23], when inserting the screws [24]. Our study found one neurological lesion in the CRPF technique with a full recovery after changing the length of the malpositioned screws and one for the ORIF technique with a permanent L5 root palsy requiring an orthosis.

The CRPF method has well-recognized risks of nerve injury [25] but when performed with high-quality image intensification, the complication rates have been low [5, 7]. According to Giannoudis et al. [8], the risk of neurological damage in the CRPF technique was estimated between 0.5 and $7.7 \%$.

Neurological assessment for pelvic fractures is essential but often difficult to perform in a polytrauma patient in the emergency room. Particular attention should be paid to the lumbosacral plexus (especially L5), the upper gluteal nerve and the pudendal nerve [25].

\section{Operative time and length of hospitalization}

A comparison of operative time for CRPF versus ORIF technique is difficult due to the diverse types of pelvic ring injuries (non-versus displaced anterior or posterior pelvic ring fractures). In our study, the operative time is practically similar with an average of 139 min for the CRPF method versus 138 min for the ORIF method. In other studies, like Giannoudis et al. and Routt et al. the percutaneous technique shows a reduced operative time [8, 22]. In the Shuler et al. study, the mean operative time was of $52 \mathrm{~min}$ in $35 \%$ of the patients with only percutaneous screws without additional procedures [23]. However, the overall range was of $170 \mathrm{~min}$.

No studies have compared the length of hospital stay between the two techniques. We found a median hospital stay of 23 days for the CRPF group versus a median hospital stay of 25 days for the ORIF group. The days of hospital stay for acute care were shorten by the transfer to other centers or to the clinical rehabilitation. Severe polytrauma patients had longer length of stay in both groups.

\section{Fluoroscopic and 0-Arm}

Screws can be inserted via an open approach in the prone position, or percutaneously in the supine position. The CRPF procedure is considered to be technically challenging and is associated with high screw malpositioning rates of up to $24 \%$ [26]. The first report of an open screw fixation of a sacroiliac dislocation was by Lehmann et al. (1934) [27]. Since then, the technique has undergone several modifications, based on Matta's widely established and standardized fluoroscopic projections (inlet and outlet) to evaluate the pelvic 
ring lesion and control the screw placement intraoperatively [25]. Due to the limitations of 2D-fluoroscopic images, a repetitive change of the $\mathrm{c}$-Arm projection $\left(90^{\circ}\right.$ to another) is mandatory to visualize the guide wire position in all three dimensions during drilling. Furthermore, the experience of the surgeon in pelvic surgery and in navigation procedures is an important factor, which may further influence these parameters. As the experience of our team increased with the $\mathrm{O}$-Arm, the operative timing decreased. It is also important to utilize a drill-tip guide wire as opposed to threaded. Further, important to advance the drill bit on oscillate and tap the bone to ensure the tip is in bone the entire way, i.e., and not out the sacral ala, in the foramen or in the sacral canal. Sometimes, proprioceptive manual feedback is difficult in patients with poor bone stock.

We have lately abandoned the use of the O-Arm because of the excessive long time for positioning of the patient and time spent to acquire intraoperative images. Instead, we are now using two fluoroscopies (inlet and outlet) during introduction of the guide wire with antero-posterior and lateral views control before inserting the cannulated screw [28].

The main limitation of this study is the small number of patients analyzed, faced in previous literature, which allows us only to affirm a decrease in intraoperative bleeding with CRPF method. There was no statistically significant difference between both techniques for the functional outcomes, the rate of infection, neurological injury, the operative time and the hospital stay.

\section{Conclusion}

Closed reduction and percutaneous iliosacral screw fixation is a potential treatment for posterior pelvic ring injuries including minimally displaced SI joint dislocations, vertical sacral fractures and/or SI joint fracture-dislocations.

This technique has lately gained in popularity for its benefits including shorter operative time, decreased blood loss, lower infection risk, and ease of anterior surgery when indicated. Moreover, this method has increased interest and familiarity among early career orthopedic trauma surgeons.

In our study we found more than $70 \%$ of good to excellent results using the Majeed's score without a better quality of reduction compared to the ORIF technique and a statistically decrease of blood loss.

However, we could not affirm a clear superiority of this technique over the ORIF for functional results, neurologic lesions, infection rates, operative time and hospital stay.

We will need larger comparative studies in the future before the standardization of the percutaneous technique for unstable pelvic ring injuries.

\section{Compliance with ethical standards}

Conflict of interest All authors declare that they have no conflict of interest.

Ethical approval The ethics committee approved the study (201702091).

Human and animal rights All procedures performed in studies involving human participants were in accordance with the ethical standards of the institutional and/or national research committee and with the 1964 Helsinki Declaration and its later amendments or comparable ethical standards.

Informed consent Informed consent was obtained from all individual participants included in the study.

\section{References}

1. Suzuki T, Shindo M, Soma K, Minehara H, Nakamura K, Uchino M, Itoman N (2007) Long-term functional outcome after unstable pelvic ring fracture. J Trauma 63:884-888

2. Tile M (1988) Pelvic ring fracture: should they be fixed? J Bone and Joint Surg. J Bone Jt Surg 70(1):1-12

3. Failinger MS, McGanity PL (1992) Unstable fractures of the pelvic ring. J Bone Jt Surg Am 74:781-791

4. Kellam JF, McMurtry RY, Paley D, Tile M (1987) The unstable pelvic fracture: operative treatment. Orthop Clin N Am 18:25-41

5. Keating JF, Werier J, Blachut P, Broekhuyse H, Meek RN, O'Brien PJ (1999) Early fixation of the vertically unstable pelvis: the role of iliosacral screw fixation of the posterior lesion. J Orthop Trauma 13(2):107-113

6. Routt M, Meier M, Kregor P (1993) Percutaneous iliosacral screws with the patient supine technique. Op Tech Orthop 3:35-45

7. Routt ML Jr, Kregor PJ, Simonian PT, Mayo KA (1995) Early results of percutaneous iliosacral screws placed with the patient in the supine position. J Orthop Trauma 9:207-214

8. Giannoudis PV, Tzioupis CC, Pape HC, Roberts CS (2007) Percutaneous fixation of the pelvic ring: an update. J Bone Jt Surg Br 89(2):145-154

9. Schweitzer D, Zylberberg A, Cordova M, Gonzalez J (2008) Closed reduction and iliosacral percutaneous fixation of unstable pelvic ring fractures. Injury 39(8):869-874

10. Majeed SA (1989) Grading the outcome of pelvic fractures. J Bone Jt Surg Br 71:304-306

11. Pohlemann T, Gänsslen A, Schellwald O, Culemann U, Tscherne H (1996) Outcome after pelvic injuries. Unfallchirurg 99(4):249-259

12. Henderson RC (1989) The long-term results of nonoperatively treated major pelvic disruptions. J Orthop Trauma 3(1):41-47

13. Gire JD, Jiang SY, Gardner MJ, Bishop JA (2018) Percutaneous versus open treatment of posterior pelvic ring injuries: changes in practice patterns over time. J Orthop Trauma 32(9):457-460

14. Routt MLC, Simonian PT (1996) Closed reduction and percutaneous skeletal fixation of sacral fractures. Clin Orthop 329:121-128

15. Elzohairy MM, Salama AM (2007) Open reduction internal fixation versus percutaneous iliosacral screw fixation for unstable posterior pelvic ring disruptions. Orthop Traumatol Surg Res 103:223-227

16. Matta JM, Saucedo T (1998) Internal fixation of pelvic ring injuries. Clin Orthop 242:83-97

17. Mullis BH, Sagi CH (2008) Minimum 1-year follow-up for patients with vertical shear sacroiliac joint dislocations treated 
with iliosacral screws: Does joint ankylosis or anatomic reduction contribute to functional outcome? J Orthop Trauma 22:293-298

18. Pohlemann T, Gänsslen A, Schellwald O, Culemann U, Tscherne $\mathrm{H}$ (1996) Outcome evaluation after unstable injuries of the pelvic ring. Unfallchirurg 99:249-259

19. Dujardin FH, Hossenbaccus M, Duparc F, Biga N, Thomine JM (1998) Long-term functional prognosis of posterior injuries in high energy pelvic disruption. J Orthop Trauma 12:145-151

20. Kokubo Y, Oki H, Sugita D et al (2017) Functional outcome of patients with unstable pelvic ring fracture. J Orthop Surg (Hong Kong) 25:2309499016684322

21. Barei DP, Bellabarba C, Mills WJ, Routt Jr. ME (2001) Percutaneous management of unstable pelvic ring disruptions. Injury 32(Suppl 1):SA33-44

22. Routt ML, Nork SE, Mills WJ (2000) Percutaneous fixation of pelvic ring disruptions. Clin Orthop Relat Res 375:15-29

23. Shuler T, Boone D, Gruen G (1995) Percutaneous iliosacral screw fixation: early treatment for unstable posterior pelvic ring disruptions. J Trauma 38:453-458

24. Templeman D, Schmidt A, Freese J, Weisman I (1996) Proximity of iliosacral screws to neurovascular structures after internal fixation. Clin Orthop 329:194-198
25. Routt ML Jr, Simonian PT, Mills WJ (1997) Iliosacral screw fixation: early complications of the percutaneous technique. J Orthop Trauma 11(8):584-589

26. Tonetti J, Carrat L, Blendea S, Troccaz J, Merloz LS et al (2001) Clinical validation of computer assisted pelvic surgery using ultrasound. A percutaneous safe technique with low radiation exposure. Stud Health Technol Inform 81:515-520

27. Lehmann J (1934) Dislocation of a hemiplevis. Zentralbl Chir $37: 2149-2152$

28. Tonetti J, Van Overschelde J, Sadok B, Vouaillat H, Eid A (2013) Percutaneous ilio-sacral screw insertion. fluoroscopic techniques. Orthop Traumatol Surg Res 8:965-972

Publisher's Note Springer Nature remains neutral with regard to jurisdictional claims in published maps and institutional affiliations. 